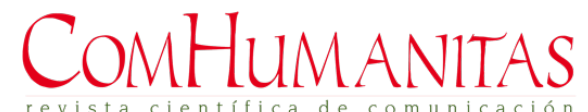

Revista ComHumanitas, ISSN: 1390-776X Vol 10, núm. 2 (2019), Mayo-agosto 2019 DOI: https://doi.org/10.31207/rch.v10i2.209

\title{
Publicidad de contenidos y su relación con el engagement de publicaciones en Facebook de tiendas de videojuegos de Quito durante el primer semestre de 2018
}

\section{Content Advertising and its relation with the engagement of publications in Facebook of videogame stores in Quito during the first semester of 2018 \\ Publicidade de conteúdos e sua relação com o engajamento de publicações no Facebook de lojas de videogame de Quito durante o primeiro semestre de 2018}

\author{
Alejandro Javier Castillo Díaz ${ }^{1}$ \\ Instituto Tecnológico Superior Cordillera (Ecuador) \\ alejandro.castillo@cordillera.edu.ec \\ Jonathan Marcelo Vinueza Suárez ${ }^{2}$ \\ Instituto Tecnológico Superior Cordillera (Ecuador) \\ jonathan.vinueza@cordillera.edu.ec
}

Fecha de recepción: 01 de abril de 2019

Fecha de recepción evaluador: 16 de abril de 2019

Fecha de recepción corrección: 01 de mayo de 2019

\footnotetext{
${ }^{1}$ Alejandro Castillo es Publicista y Magister en Dirección de Comunicación, con 13 años de experiencia en publicidad y marketing. Propietario de Castelvania S.A., agencia de manejo de marcas en espacios web 2.0. Experiencia en la Docencia Superior de Marketing, Publicidad y Comunicación. ORCID: https://orcid.org/0000-0002-7132-6265

${ }^{2}$ Jonathan Vinueza es Licenciado en Comunicación Social, con experiencia en Docencia en la carrera de Diseño Gráfico en el Instituto Tecnológico Superior Cordillera. Ha trabajado en Wepa Music Records como Diseñador Multimedia. Maestrante de Diseño Gráfico Digital en la Universidad de la Rioja (España). ORCID: https://orcid.org/0000-0001-6471-1344
} 


\title{
Resumen
}

En el presente estudio se analizó el engagement de las 30 tiendas de videojuegos de Quito que poseen una página en Facebook y que realizaron al menos una publicación en esta red social durante el primer semestre de 2018. El objetivo fue comprobar si la utilización de publicidad de contenidos influyó en esta métrica. Para ello, se clasificó a las tiendas en dos grupos, 17 que realizaron publicidad de contenidos entre Enero y Junio de 2018 y 13 que no lo hicieron. Se realizó una observación de cada fanpage para determinar datos como el número de seguidores de las tiendas, las interacciones de cada una de sus publicaciones y finalmente su engagement. Se demostró que la utilización de publicidad de contenidos de las tiendas del primer grupo, influyeron en los niveles de engagement con respecto a las tiendas del segundo grupo. Por lo tanto, se visualiza la importancia de incorporar a comunicadores visuales expertos en la generación de publicaciones de valor para medios sociales en esta industria de entretenimiento.

Palabras clave: Redes sociales, videojuegos, métricas en Facebook, interacciones, comunicación visual, marketing digital

\begin{abstract}
In this study, the engagement of the 30 videogame stores in Quito that have a Facebook page and made at least one publication in this social network was analized during the first semester of 2018. The objective was to check if the use of advertising content in each page influenced its metric. To do this, the stores were classified into two groups, 17 that made advertising content between January and June 2018 and 13 that did not. Each fanpage was analyzed to determine data such as the number of followers of the stores, the interactions of each of their publications and finally their engagement. The study demonstrated that the use of advertising content from the stores of the first group, influenced the levels of engagement with respect to the stores of the second group. Therefore, it is demonstrated the importance of incorporate visual communicators experts in the generation of valuable publications for social media in this entertainment industry.
\end{abstract}

Keywords: Social network, videogames, Facebook metrics, interactions, visual comunication, digital marketing

\section{Resumo}

No presente estudo foi analisado o engagement das 30 lojas de vídeo game em Quito que tem uma página no Facebook e que fez pelo menos uma publicação nesta rede social durante o primeiro semestre do 2018. O objetivo foi verificar se o uso de publicidade de conteúdos influenciaram nesta métrica. Para fazer isso, as lojas foram classificadas em dois grupos, 17 que anunciaram conteúdo entre janeiro e junho de 2018 e 13 que não o fizeram. Uma observação de cada fanpage foi feita para determinar dados como o número de seguidores das lojas, as interações de cada uma de suas publicações e, finalmente, seu engagement. Ficou demonstrado que o uso de publicidade de conteúdo das lojas do primeiro grupo, influenciou os níveis deengagement em relação às lojas do segundo 
grupo. Portanto, é vista a importância de incorporar comunicadores visuais especializados na geração de publicações de valor para mídias sociais nesta indústria de entretenimento.

Palavras-chave: redes sociais, videogames, métricas no Facebook, interações, comunicação visual, marketing digital

\section{Introducción}

Desde la aparición de Atari Pong en 1977, los videojuegos han generado diversas polémicas y opiniones divididas acerca de si es o no recomendable que sean utilizados (Snead, 2014). En diciembre de 1993, el Senador de los Estados Unidos, Joe Liberman, expuso que los videojuegos corrompen la mente de los jóvenes, lo que propició la creación de la Junta de la Clasificación de Software de Entretenimiento, cuya primera resolución fue la de asignar la calificación M (Mature) al videojuego Mortal Kombat (El Universo, 2014). Casi 25 años después, en junio de 2018, la Organización Mundial de la Salud, declaró a la adicción a los videojuegos como una enfermedad mental (Rodríguez, 2018).

A pesar de ello, la industria ha experimentado un crecimiento continuo y son cada vez más las personas que ingresen a esta forma de entretenimiento digital. Durante 2015, los videojuegos generaron ingresos por más de 91 mil millones de dólares a nivel mundial, contra los 38.300 millones que generó la industria del cine o los 15 mil millones generados por la industria de la música ese mismo año (Veintimilla, 2018). De acuerdo con estadísticas presentadas por la Entertainment Software Association, más de 150 millones de personas en Estados Unidos juegan videojuegos y, en el 64\% de los hogares estadounidenses, se encuentra al menos una consola asignada exclusivamente para este fin (ESA, 2018). Dentro de estas estadísticas, se presenta que la industria de los videojuegos emplea a más de 220 mil personas en los 50 estados de ese país.

Algo que ha facilitado este crecimiento de la industria, es la importancia que tienen los videojuegos en la construcción de relaciones de amistad y compañerismo entre adolescentes. En España, los jóvenes que tienen acceso a videojuegos online, invierten de una a cuatro horas diarias en ellos, siendo los géneros más practicados, los que son orientados a los deportes o a la guerra (Muros, Aragón y Bustos, 2013). Un denominador común de estos juegos, es la sensación de adrenalina, producto de las estrategias realizadas para ganar una partida. Esto ha dotado a la industria de nuevos matices, en los que, en ciertos países como Corea del Sur, los videojuegos son una profesión, tanto para jugadores profesionales que compiten en los denominados e-sports, como para YouTubers que generan ingresos por la transmisión de eventos relacionados a este medio (Sinde, Medrano y Martinez de Morentin, 2015).

En Quito, capital de Ecuador, son varias las tiendas que comercializan consolas y videojuegos. El Bosque, uno de los Centros Comerciales más grandes y variados de la ciudad, cuenta con 6 tiendas de videojuegos, contra apenas 4 de artesanías y 2 jugueterías 
(Centro Comercial El Bosque, 2018). La oferta de productos de esta categoría resalta aún más en canales de compra y venta por Internet, como Mercado Libre, donde se encuentran un total de 18.861 anuncios con la etiqueta videojuegos a nivel nacional (Mercado Libre, 2018). Adicionalmente, Facebook, página web que se encuentra como la cuarta más visitada en Ecuador (Alexa, 2018), no ha sido ajena al crecimiento de oferta y demanda de estos artículos. Hasta agosto de 2018, en la ciudad de Quito, se registraban 84 tiendas de videojuegos que poseían una página en esta red social (Facebook, 2018).

En este contexto, se visualiza la necesidad de las tiendas de diferenciarse de sus competidores, a través de mecanismos publicitarios basados en contenidos que promuevan experiencias interactivas con sus usuarios (Carrillo y Castillo, 2005). Además, estos contenidos deberán estar enfocados en cambiar la percepción general de que los videojuegos corrompen a niños y jóvenes y reforzar algunas de sus aplicaciones positivas como, por ejemplo, dentro del ámbito educativo (Esnaola y Levis, 2009). El objetivo de los contenidos de valor es el de generar engagement, lo que se refiere al compromiso y participación de usuarios con un determinado medio, contenido o marca (Tur-Viñes y Rodríguez, 2014).

\section{Marco referencial}

El concepto de que las redes sociales y los videojuegos están únicamente destinados al entretenimiento, ha quedado desfasado. Las posibilidades de estos medios interactivos dentro de ámbitos sociales, educativos, culturales y laborales redefinen sus posibilidades y alcances (Muros, Aragón y Bustos, 2013). A su vez, medios tradicionales de comunicación y otras herramientas de marketing convencional, han quedado relegados en favor de los medios sociales digitales, en los que existe una comunicación bidireccional entre las organizaciones y sus públicos (Sarmiento, 2017). En este escenario, surgen mecanismos publicitarios que se basan en la interacción y en la creación de contenidos de valor para el usuario, fomentando su sentido de pertenencia y apego emocional.

A diferencia de los medios de comunicación tradicionales, los medios sociales permiten a los públicos de las organizaciones pasar de ser receptores de mensajes a ser ellos quienes difundan los mensajes emitidos por las marcas a las que siguen. La comunicación de marcas ha sido reemplazada por la comunicación de usuarios, en donde se contempla a los públicos como los primeros comunicadores de la estrategia empresarial y los promotores de los contenidos de una organización (Sarmiento, 2017).

Castillo y Carrillo (2005) conceptualizaron a la Nueva Publicidad Digital (NPD) como aquella que se fundamenta en la generación de nuevos contenidos, capaces de enriquecer la experiencia de contacto del usuario con el canal digital y lograr la fidelización de la marca. La publicidad de contenidos no busca únicamente un clic a un anuncio, sino generar experiencias interactivas a través de servicios de transmisión de información de interés de un determinado tema en diversos formatos. Los autores apuntan a la creatividad como la herramienta que permite integrar al servicio con la interactividad. 
Para Ureña, Herrera-Murillo y Rodríguez-Martínez (2014), el engagement es un estado mental de los usuarios que los inspira a disfrutar de una determinada representación, acción u objeto y que influye en los niveles de energía de los públicos, quienes se sienten motivados a dedicar tiempo a la actividad que están realizando, debido a que es disfrutada por ellos. El engagement, en redes sociales, surge como consecuencia de las publicaciones compartidas por las marcas y depende de factores como el tipo de publicación, el formato y el momento en el que se publica. Entre los indicadores para calcular el engagement de una marca en Facebook, están en el número de publicaciones en su muro, sus comentarios, la cantidad de reacciones generadas y el número de veces que las publicaciones fueron compartidas.

Paladines, Granda y Velásquez (2014), hablan de los prosumidores, los consumidores de una marca que son los responsables de crear el contenido para ella. Con esto, refieren la importancia de generar publicaciones que no sean inertes, sino que busquen la interacción y participación de los públicos de interés. También mencionan el Facebook Content, el tipo de contenidos que pretende la participación del target a través de la generación de experiencias. Adicionalmente, proponen que las marcas pueden lograr un posicionamiento deseado únicamente haciendo uso de redes sociales como medios de comunicación, mientras exista claridad en el tipo de posicionamiento que se quiere lograr y en la calidad de contenidos que se ofrece.

El objetivo del engagement en redes sociales, es el de estimular a las audiencias y el de consolidar el fenómeno fan (seguidor). Estos medios de comunicación requieren de la participación de profesionales que estén relacionados con nuevas formas narrativas. Los canales digitales ofrecen un mundo de posibilidades de interacción, además de la reproducción, reelaboración y producción de nuevos contenidos por parte de los usuarios. Además, el análisis de respuesta inmediata por parte de la audiencia de un fanpage, permite definir la línea de contenidos a utilizar por una marca para mejorar su engagement (Tur-Viñes y Rodríguez, 2014).

Revuelta y Bernabé (2012) consideran a los videojuegos como medios de comunicación interactivos. Adicionalmente, los autores plantearon que las redes sociales son los espacios idóneos para su crecimiento, por las posibilidades de convertirlos en videojuegos sociales, es decir, aquellos que permiten a los usuarios interactuar con otras personas para jugar a través de mecanismos online, o para compartir opiniones y experiencias. Facebook ofrece un nivel de interacción inalcanzable para los videojuegos por si solos, por lo que pueden adquirir nuevas dimensiones al crecer dentro de esta red social.

Esnaola y Levis (2009) realizaron un estudio para descubrir las posibilidades educativas que tienen los videojuegos en espacios web 2.0, especialmente en Facebook por ser la red social con mayor usabilidad a nivel mundial. En esta investigación, se encontró que los videojuegos poseen un gran potencial educativo dentro de las redes sociales, sobre todo aquellos que son de estrategia o simuladores situacionales. La 
generación de contenidos que sean de interés, favorece el proceso de aprendizaje de los estudiantes.

En Ecuador, no existen estudios que prueben un crecimiento de la industria de los videojuegos como producto de la utilización de medios sociales de comunicación. Sin embargo, investigaciones realizadas en el país, han demostrado la importancia de la utilización de las tecnologías de la información en la vinculación y participación de públicos con marcas, actividades y eventos de diversos sectores.

Con motivo de la gran saturación de mensajes políticos en medios sociales, producto de las elecciones presidenciales en Ecuador entre Lenin Moreno y Guillermo Lasso en 2017, Zumárraga, Reyes y Carofylis (2017), plantearon la hipótesis de que los ecuatorianos que participan activamente en grupos de opinión en redes sociales, tienen más posibilidades de vincularse a organizaciones o actividades políticas. A través de este estudio, pudieron concluir que las personas que realizan activismo político en Facebook, WhatsApp y Twitter, tienen una mayor predisposición a vincularse a partidos $\mathrm{y}$ movimientos políticos e incluso a participar en elecciones para cargos públicos que las personas que no lo hacen.

Para Punín, Martínez y Rencoret (2014), los medios digitales en Ecuador tienen deficiencias en su comunicación bidireccional y, en muchos casos, se limitan a transmitir información en sus redes sociales o páginas web, pero no buscan la interacción. Recomiendan la utilización del transmedia, entendido como el fenómeno mediático que permite al usuario interactuar con los emisores del mensaje desde de su teléfono celular, a través de contenido multimedia interactivo como aplicaciones o juegos.

\section{Metodología}

Los primeros datos que se tomaron como base para la realización del presente estudio, fueron aquellos obtenidos a través de la fase de observación las tiendas de videojuegos en Quito que poseen un fanpage en Facebook:

- Existen 84 tiendas de videojuegos en Quito que poseen una página en Facebook.

- 30 de estas tiendas han realizado al menos una publicación entre enero y junio de 2018 y se convierten, por tanto, en las tiendas analizadas para esta investigación

Como siguiente punto, se categorizaron a las tiendas de videojuegos en dos grupos:

- Grupo A: Tiendas que utilizaron publicidad de contenidos entre enero y junio de 2018

- Grupo B: Tiendas que no utilizaron publicidad de contenidos entre enero y junio de 2018 
Para realizar esta categorización, se tomaron como fuente los apuntes realizados por Carrillo y Castillo (2005), que definen a la publicidad de contenidos en medios digitales, como aquella que promueve la interacción del público con la marca y aporta información y datos relevantes que generen experiencias. Por lo tanto, las tiendas categorizadas dentro del grupo A, fueron aquellas que, durante el periodo establecido, realizaron al menos una publicación que haya buscado la conversación y participación de sus seguidores u otorgarles datos relevantes sobre la industria o algún videojuego en particular. Las tiendas categorizadas dentro del grupo B, fueron aquellas que únicamente proporcionaron información sobre los productos que comercializaban y sus precios, sin profundizar en mensajes que generen la vinculación emocional de sus usuarios con la marca. Cada página fue codificada de acuerdo con el grupo al que pertenece para el procesamiento de su información.

A continuación, se procedió a determinar si las páginas de las 30 tiendas analizadas en este estudio son administradas por sus dueños o si recurren a expertos de comunicación visual para hacerlo. Para ello, se entabló una conversación por mensajería instantánea en Facebook con cada una de estas páginas, lo que permitió, además, medir si existe una respuesta eficiente y oportuna por esta vía.

Una vez levantada esta información, se procedió a calcular el engagement de todas las páginas de las tiendas de los grupos A y B. Para ello, basados en los estudios de este fenómeno de Ureña, Herrera-Murillo y Rodríguez-Martínez (2014) y de Tur-Viñes y Rodríguez (2014), se contabilizaron los siguientes datos de cada una de las 30 tiendas en el periodo de enero a junio de 2018 :

- Número de seguidores

- Número de publicaciones realizadas en Facebook

- Número total de reacciones en publicaciones

- Número total de comentarios en publicaciones

- Número total de publicaciones compartidas

Dentro de una red social, el engagement es un valor porcentual que se basa en el nivel de participación del total de seguidores de una página con una publicación en Facebook. Para encontrar este dato, se requiere conocer el número interacciones que obtuvo la publicación, que es la suma de sus reacciones, comentarios y compartidos (Núñez, 2017). Con estos valores, se calculó el engagement a través de la siguiente fórmula:

Engagement $=$ Interacciones $* 100 /$ Número de seguidores de la página

Para el presente estudio, lo que se buscó es obtener el engagement total del periodo entre enero y junio de 2018, para generar cuadros comparativos entre las tiendas analizadas el grupo A con respecto a las tiendas analizadas del grupo B y así, determinar 
si se cumplió o no la hipótesis. Para este efecto, se procedió a obtener el número total de interacciones del periodo a través de la suma del total de reacciones, comentarios y compartidos de todas las publicaciones durante el primer semestre del año 2018 por cada tienda analizada. Este valor fue dividido para el número de publicaciones realizadas en las páginas, lo que permitió obtener su número promedio de interacciones $\mathrm{y}$, posteriormente su engagement de acuerdo con la siguiente operación:

Engagement del periodo $=$ Promedio de interacciones $* 100 /$ Número de seguidores de la página

\section{Análisis de datos}

El grupo al que pertenecen las páginas de Facebook y el perfil que tienen los administradores de los fanpage, son los primeros resultados finales de esta fase de la investigación. Se generaron, por tanto, tablas comparativas de valores totales y porcentuales de cada uno de ellos.

Todos los datos recopilados en el presente estudio fueron ingresados en una hoja de del programa Microsoft Excel 2013, lo que permitió obtener los valores calculados a través de operaciones aritméticas. Las celdas ingresadas en el software fueron las siguientes:

- Código de página

- Número de publicaciones de enero a junio de 2018

- Número de seguidores

- Número de reacciones

- Número de comentarios

- Número de compartidos

- Número total de interacciones

- Promedio de interacciones

- Cálculo del engagement

Esta tabla es presentada en la sección de resultados, junto con otras que evidencian cuáles son las cinco páginas que presentan valores más altos de las siguientes variables:

- Seguidores

- Interacciones

- Engagement 
Los valores del engagement de cada página, junto con el grupo en el que está categorizado, fueron ingresados en el software IBM SPSS Statistics 23. Como primer paso, se realizó una prueba de normalidad de Shapiro-Wilk ya que la muestra es $n<50$. Se generaron, también, tablas comparativas de las tiendas de grupo A con respecto a las tiendas del grupo B de los siguientes estadísticos descriptivos:

- Media

- Moda

- Mediana

- Desviación típica

- Varianza

Finalmente, para determinar si las diferencias son significativas, se aplicó la prueba paramétrica $T$ de variables independientes del engagement.

\section{Hallazgos}

La revisión de las 30 páginas de videojuegos que han realizado publicaciones entre enero y junio de 2018, permitió visualizar que 17 de ellas realizaron publicidad de contenidos durante el periodo, mientras que 13 páginas no lo hicieron como se visualiza a continuación (Tabla 1):

Tabla 1: Categorización de tiendas de consolas y videojuegos

\begin{tabular}{llll}
\hline Código & No. tiendas & Porcentaje & Descripción \\
\hline Grupo A & 17 & $56,67 \%$ & $\begin{array}{l}\text { Realizaron publicidad de contenidos entre } \\
\text { Enero y Junio de } 2018\end{array}$ \\
\hline Grupo B & 13 & $43,33 \%$ & $\begin{array}{l}\text { No realizaron publicidad de contenidos entre } \\
\text { Enero y Junio de } 2018\end{array}$ \\
& & &
\end{tabular}

Para organizar correctamente la información con el fin de establecer cuadros comparativos, cada página fue codificada de acuerdo con el grupo al que pertenece (Tabla 2):

Tabla 2: Codificación de páginas de tiendas de consolas y videojuegos

\begin{tabular}{ll}
\hline Códigos de páginas & Descripción \\
\hline A1 - A17 & Páginas de tiendas de consolas y videojuegos que pertenecen al grupo A \\
\hline B1 - B13 & Páginas de tiendas de consolas y videojuegos que pertenecen al grupo B \\
\hline
\end{tabular}


De las 30 páginas estudiadas, seis de ellas (20\%) facilitaron información del perfil del administrador del fanpage, mientras que las otras 24 (80\%) no proporcionaron estos datos. Los resultados obtenidos fueron que el $50 \%$ de los administradores de las tiendas manejan sus propios fanpage y el otro 50\% recurre a profesionales del diseño gráfico para este fin (Tabla 3):

Tabla 3: Categorización de los administradores de los fanpage de las tiendas de videojuegos estudiadas

\begin{tabular}{llll}
\hline $\begin{array}{l}\text { No. } \\
\text { tiendas }\end{array}$ & Porcentaje & Códigos de páginas & Descripción \\
\hline 3 & $50 \%$ & $7 \mathrm{~A}, 8 \mathrm{~A}, 9 \mathrm{~B}$ & Los fanpage son manejados por el administrador de la tienda \\
\hline 3 & $50 \%$ & $1 \mathrm{~A}, 5 \mathrm{~A}, 11 \mathrm{~A}$ & Los fanpage son manejados por un diseñador gráfico contratado
\end{tabular}

Un dato relevante de esta conversación entablada es que cinco de las seis tiendas que proporcionaron datos para el estudio $(83,33 \%)$ fueron categorizadas dentro del grupo A y apenas una de ellas $(16,67 \%)$ fue categorizada en el grupo B.

Tras la categorización de los fanpage, se presenta la contabilización de los datos de cada una de las publicaciones de las páginas analizadas, así como el cálculo del engagement en el periodo transcurrido entre enero y junio de 2018 (Tabla 4):

Tabla 4: Datos estadísticos de las publicaciones en los fanpage

\begin{tabular}{|c|c|c|c|c|c|c|c|c|}
\hline $\begin{array}{l}\text { Código } \\
\text { página }\end{array}$ & $\begin{array}{l}\text { Publicaciones } \\
\text { de enero a } \\
\text { junio } 2018\end{array}$ & $\begin{array}{l}\text { Número de } \\
\text { seguidores }\end{array}$ & Reacciones & Comentarios & Compartidos & Interacciones & $\begin{array}{l}\text { Promedio } \\
\text { interacciones }\end{array}$ & Engagement \\
\hline A1 & 9 & 121 & 44 & 2 & 7 & 53 & 5,89 & 4,87 \\
\hline A2 & 40 & 21,127 & 34795 & 892 & 972 & 36659 & 916,48 & 4,34 \\
\hline A3 & 21 & 147 & 127 & 0 & 4 & 131 & 6,24 & 4,24 \\
\hline A4 & 19 & 37 & 26 & 1 & 0 & 27 & 1,42 & 3,84 \\
\hline A5 & 91 & 1,653 & 4429 & 129 & 457 & 5015 & 55,11 & 3,33 \\
\hline A6 & 117 & 1,394 & 1205 & 428 & 2203 & 3836 & 32,79 & 2,35 \\
\hline A7 & 22 & 312 & 94 & 12 & 15 & 121 & 5,50 & 1,76 \\
\hline A8 & 18 & 1,669 & 348 & 56 & 42 & 446 & 24,78 & 1,48 \\
\hline A9 & 5 & 879 & 28 & 11 & 6 & 45 & 9,00 & 1,02 \\
\hline A10 & 90 & 4,318 & 2198 & 347 & 412 & 2957 & 32,86 & 0,76 \\
\hline A11 & 154 & 5,361 & 3278 & 376 & 2114 & 5768 & 37,45 & 0,70 \\
\hline A12 & 51 & 3,869 & 640 & 26 & 223 & 889 & 17,43 & 0,45 \\
\hline A13 & 162 & 10,255 & 2629 & 665 & 1002 & 4296 & 26,52 & 0,26 \\
\hline A14 & 106 & 3,005 & 739 & 31 & 19 & 789 & 7,44 & 0,25 \\
\hline A15 & 9 & 747 & 9 & 1 & 0 & 10 & 1,11 & 0,15 \\
\hline A16 & 127 & 10,324 & 380 & 9 & 14 & 403 & 3,17 & 0,03 \\
\hline A17 & 402 & 48,832 & 1487 & 389 & 2976 & 4852 & 12,07 & 0,02 \\
\hline B1 & 2 & 42 & 2 & 0 & 0 & 2 & 1,00 & 2,38 \\
\hline B2 & 8 & 527 & 11 & 3 & 25 & 39 & 4,88 & 0,93 \\
\hline
\end{tabular}




\begin{tabular}{|c|c|c|c|c|c|c|c|c|}
\hline B3 & 19 & 1,103 & 88 & 29 & 76 & 193 & 10,16 & 0,92 \\
\hline B4 & 1 & 438 & 1 & 0 & 2 & 3 & 3,00 & 0,68 \\
\hline B5 & 4 & 17,703 & 368 & 31 & 7 & 406 & 101,50 & 0,57 \\
\hline B6 & 3 & 77 & 1 & 0 & 0 & 1 & 0,33 & 0,43 \\
\hline B7 & 2 & 169 & 1 & 0 & 0 & 1 & 0,50 & 0,30 \\
\hline B8 & 84 & 89,293 & 11026 & 1043 & 377 & 12446 & 148,17 & 0,17 \\
\hline B9 & 9 & 77 & 1 & 0 & 0 & 1 & 0,11 & 0,14 \\
\hline $\mathrm{B} 10$ & 15 & 4,402 & 58 & 10 & 13 & 81 & 5,40 & 0,12 \\
\hline B11 & 21 & 1,268 & 20 & 4 & 2 & 26 & 1,24 & 0,10 \\
\hline B12 & 2 & 736 & 1 & 0 & 0 & 1 & 0,50 & 0,07 \\
\hline B13 & 26 & 1,230 & 17 & 2 & 1 & 20 & 0,77 & 0,06 \\
\hline
\end{tabular}

Se evidencian diferencias en los datos estadísticos de publicaciones entre páginas del mismo grupo al que pertenecen. Para encontrar si existe una tendencia que demuestre que el realizar publicidad de contenidos afecta de forma positiva de las publicaciones de los fanpage de tiendas de consolas y videojuegos en la ciudad de Quito, se presentan tablas de las 5 páginas que mejores resultados obtuvieron con respecto a las variables seguidores, interacciones y cálculo de engagement (Tablas 5, 6 y 7):

Tabla 5: Top 5 de las páginas con mayor número de seguidores

\begin{tabular}{ll}
\hline Código Página & Número de seguidores \\
\hline B8 & 89,293 \\
\hline A17 & 48,832 \\
\hline A2 & 21,127 \\
\hline B5 & 17,703 \\
\hline A16 & 10,324 \\
\hline
\end{tabular}

Tres de las cinco páginas que aparecen entre los fanpage con mayor número de seguidores $(60 \%)$ pertenecen a tiendas que realizaron publicidad de contenidos entre el periodo comprendido entre enero y junio de 2018 mientras que dos de ellas (40\%) pertenecen a tiendas que no lo hicieron.

Tabla 6: Top 5 de las páginas con mayor número de interacciones

\begin{tabular}{ll} 
Código de página & Número de interacciones \\
\hline A2 & 36,659 \\
\hline B8 & 12,446 \\
\hline A11 & 5,768 \\
\hline A5 & 5,015 \\
\hline A17 & 4,852 \\
\hline
\end{tabular}


En este caso, se puede evidenciar una tendencia favorable hacia las páginas del grupo A con relación a las páginas del grupo $\mathrm{B}$, ya que cuatro de las cinco páginas con mayor número de interacciones durante el periodo (80\%) pertenecen a este grupo. Otro dato relevante, es que tres de las páginas de estas tiendas (B8, A5 y A17), aparecen en las dos listas.

Tabla 7: Top 5 de las páginas con mayor engagement

\begin{tabular}{ll}
\hline Código de página & Engagement \\
\hline A1 & 4,87 \\
\hline A2 & 4,34 \\
\hline A3 & 4,24 \\
\hline A4 & 3,84 \\
\hline A5 & 3,33 \\
\hline
\end{tabular}

Con respecto al engagement, las cinco tiendas que figuran con el valor más alto de esta variable pertenecen al grupo A, lo que muestra una incidencia de la utilización de publicidad de contenidos en la afectación de este valor. Salta a la vista que, dentro de las tiendas analizadas, la tienda A2 estuvo presente en todas las tablas (mayor número de seguidores, más interacciones, mayor engagement), mientras que la tienda con código A5 estuvo presente en dos de ellas.

La prueba de normalidad de Shapiro-Wilk, comprueba que los datos del engagement de ambos grupos son normales $(p>, 001)$, como se detalla en la siguiente tabla (Tabla 8):

Tabla 8: Prueba de normalidad Shapiro-Wilk de las variables de ambos grupos

\begin{tabular}{lcccc}
\hline & & \multicolumn{3}{c}{ Shapiro-Wilk } \\
\cline { 3 - 5 } & Grupo & Estadístico & Gl & Sig. \\
\hline \multirow{2}{*}{ Engagement } & Grupo A &, 855 & 17 &, 013 \\
& Grupo B &, 726 & 13 &, 001 \\
\hline
\end{tabular}

A continuación, se establecen tablas comparativas de la media, moda, mediana, deviación típica y varianza del engagement de las tiendas del grupo A con respecto a las tiendas del grupo B (Tabla 9):

Tabla 9: Estadísticos descriptivos del engagement de ambos grupos

\begin{tabular}{lrr}
\hline & Grupo A & Grupo B \\
\hline Media & 1,7559 &, 5285 \\
\hline Mediana & 1,0200 &, 3000 \\
\hline Moda &, $02^{\mathrm{a}}$ &, $06^{\mathrm{a}}$ \\
\hline Desviación estándar & 1,71804 &, 63828 \\
\hline Varianza & 2,952 &, 407 \\
\hline
\end{tabular}

Existen múltiples modos. Se muestra el valor más pequeño 
Se puede apreciar que los valores de media y mediana del engagement del grupo A, son superiores a los valores de estos estadísticos del grupo B.

Tomando en cuenta que la distribución es normal, se requiere la utilización de una prueba paramétrica para comprobar si las diferencias entre ambos grupos son significativas. Por tanto, se utilizó la prueba $T$ para muestras independientes para lo cual, se plantearon 2 posibles hipótesis:

$H_{0}=$ La utilización de publicidad de contenidos en Facebook por parte de tiendas de consolas y videojuegos de la ciudad de Quito en el primer semestre de 2018, no influyó en el engagement de sus publicaciones

$H^{l}=$ La utilización de publicidad de contenidos en Facebook por parte de tiendas de consolas y videojuegos de la ciudad de Quito en el primer semestre de 2018, si influyó en el engagement de sus publicaciones.

La prueba de Levene de igualdad de varianzas es menor a $0,05(p=, 000)$, por lo que se asume que las varianzas son distintas. La significación asintótica bilateral de $T$ es menor a $0,05(p=, 013)$ para el fenómeno estudiado, con lo que queda rechazada la hipótesis nula. Se acepta, entonces, que el engagement es distinto en grupos de tiendas de videojuegos que utilizan publicidad de contenidos en Facebook con respecto a las que no lo hacen.

\section{Discusión}

Para el presente estudio, se ha definido al engagement como el gran objetivo de las publicaciones en una red social, al involucrar a otras métricas como los seguidores y las interacciones (Núñez, 2017). La prueba $T$ de muestras independientes, demuestra que la publicidad de contenidos afecta a los niveles de este fenómeno, pero, a su vez, hay otras lecturas que se extraen de los resultados de la investigación.

Por un lado, pese a que no se han podido encontrar referencias bibliográficas que vinculen a la industria del diseño gráfico con la generación de contenidos de valor para redes sociales, de seis tiendas que facilitaron información sobre quiénes administran sus páginas de Facebook, el 50\% eran manejadas por diseñadores gráficos. Esto puede obedecer a la importancia que tiene la industria gráfica en la generación y gestión de marcas (Hidalgo, Chiriboga, y Mora, 2015). Cada vez existen más empresas ecuatorianas que destinan presupuestos para la generación de espacios en medios sociales (Paladines, Granda, y Velásquez, 2014), por lo que diseñadores especializados en la administración de este tipo de canales, pueden verse favorecidos en un campo laboral que se encuentra en pleno crecimiento.

Por otro lado, al observar que apenas 30 de las 84 tiendas de este sector que poseen una página en Facebook han realizado al menos una publicación durante el periodo estipulado, se pueden corroborar las apreciaciones realizadas por Punín, Martínez y Rencoret (2014). Los autores determinaron que en Ecuador no se ha trabajado de manera correcta en estrategias de marketing y publicidad que conviertan a las nuevas plataformas 
digitales en vehículos de comunicación interactivos, desaprovechando las potencialidades sociales de estos medios.

La publicidad de contenidos, entendida como aquella que pretende la creación de un vínculo emocional entre la marca y sus públicos (Carrillo y Castillo, 2005), resulta fundamental a la hora de buscar que los usuarios se conviertan, a través de su opinión, en los principales comunicadores de la marca y los gestores de su imagen (Sarmiento, 2017). Al conocer que el 56,67\% de las tiendas que realizaron publicaciones entre enero y junio de 2018, recurrieron a contenidos de valor que las favorezcan a un acercamiento emocional con sus públicos (Tabla 1), se pueden realizar las siguientes interpretaciones:

1. Si bien, el número de tiendas que realizaron publicaciones durante el periodo estudiado son una minoría, la mayoría de las que lo hicieron tienen presente las potencialidades sociales de estos medios interactivos y buscan una comunicación integradora que genere vínculos emocionales entre la marca y sus públicos.

2. Más del $40 \%$ de las tiendas estudiadas no han recurrido a contenidos de valor, limitándose únicamente a promoción de sus productos, por lo que se puede evidenciar que no existe un consenso en la estrategia que debe seguir una tienda de videojuegos en Facebook.

Otros datos de gran relevancia, se los puede extraer de los rankings de las páginas estudiadas (Tablas 5, 6 y 7). En ellos, se pudo constatar que en el top 5 de las páginas con mayor número de seguidores, aparecieron dos que pertenecían al grupo $\mathrm{B}$, es decir al grupo de tiendas que no realizaron publicidad de contenidos durante el periodo; mientras que, en el ranking del engagement, las cinco tiendas que aparecieron en el top, pertenecían al grupo A. Además, solo una tienda apareció en ambos rankings. Esto pone en evidencia que, el tener un alto número de seguidores no significa necesariamente el poseer un engagement ideal, por lo que las marcas deben priorizar sus esfuerzos en generar una estrategia de comunicación que les permita generar vínculos emocionales con sus fans y no únicamente una gran cantidad de ellos.

Los niveles ideales de engagement en una publicación, son aquellos que oscilan entre el 0,5 y el 1\% (Barud, 2017). De las 17 páginas que utilizaron publicidad de contenidos en el primer semestre de 2018, el 64,7\% llegaron o superaron esos niveles. En cambio, apenas un $38,46 \%$ de las que tiendas que no utilizaron contenidos de valor en sus publicaciones durante el mismo periodo, alcanzaron lo que se puede denominar un engagement óptimo. Existen, por tanto, diferencias muy marcadas entre ambos grupos de tiendas, siendo la publicidad de contenidos la única variable bajo la cual se categorizaron ambos tipos de negocio.

El engagement motiva a los públicos a que se relacionen con una marca y fortalezcan su vínculo emocional con ella (Tur-Viñes y Rodríguez, 2014). Al aceptar la hipótesis de que la publicidad de contenidos favorece este fenómeno (Tabla 10), la generación de publicaciones que promuevan a los videojuegos como instrumentos 
interactivos con aplicaciones positivas (Esnaola y Levis, 2009), puede ser el vehículo que permita legitimarlos y fomentar su crecimiento en Ecuador.

Finalmente, se visualiza la importancia de la presencia de diseñadores gráficos en el proceso de consolidación de esta forma de entretenimiento (Tabla 3). Existe la necesidad de contar con profesionales de la comunicación visual preparados y capacitados, no solo en el uso adecuado de herramientas informáticas, sino también en la gestión de contenidos de valor para tiendas de consolas y videojuegos en Quito. Se requiere, que este talento humano promueva la interacción con los prosumidores que siguen a las marcas que pertenecen a esta industria de entretenimiento y así favorecer a su aceptación y crecimiento en el país.

\section{Conclusiones}

Con la presentación de resultados del presente estudio, se concluye que:

- Durante el primer semestre del año 2018,30 de las 80 tiendas que poseían una página en Facebook realizaron publicaciones en su fanpage.

- De estas 30 tiendas, 17 realizaron publicidad de contenidos entre enero y junio de 2018 mientras que 13 no lo hicieron.

- Tan solo 6 de las 30 tiendas facilitaron información sobre quiénes administraban sus páginas. El resultado obtenido, fue que el 50\% de ellas eran administradas por sus dueños y el otro $50 \%$ por diseñadores gráficos contratados para ese fin.

- 16 de las tiendas que fueron estudiadas cumplían con niveles ideales de engagement. El principal motivo que influyó para este fenómeno fue el de la utilización de publicidad de contenidos. Se determinó, además, que otros aspectos como el número de interacciones o seguidores de las tiendas no afectaron en estos valores.

- Los resultados comparativos probaron diferencias significativas entre los grupos de tiendas de ambos grupos, favoreciendo a los niveles de engagement de los negocios que utilizaron publicidad de contenidos durante el periodo estudiado.

\section{Bibliografía}

Alexa. (Agosto de 2018). Top Sites in Ecuador. Recuperado el 25 de Agosto de 2018, de https://www.alexa.com/topsites/countries/EC

Barud, S. (30 de Junio de 2017). Interacción en Facebook: ¿Cuáles son los niveles ideales? Recuperado el 9 de Febrero de 2019, de Agora Pulse: https://www.agorapulse.com/es/blog/interaccion-en-facebook-niveles-ideales 
Carrillo, M. V., y Castillo, A. (Junio a Julio de 2005). La Nueva Publicidad Digital (NPD): Servicios Digitales y Contenidos Interactivos que Generen Experiencias en los Consumidores. Razón y Palabra(45). Recuperado el 25 de Agosto de 2018, de http://www.redalyc.org/html/1995/199520623004/

Centro Comercial El Bosque. (2018). Shopping. Quito, Pichincha, Ecuador. Recuperado el 25 de Agosto de 2018, de http://www.elbosque.com.ec/shopping.html

El Universo. (5 de Junio de 2014). Mortal Kombat cambió la industria de videojuego. Guayaquil, Guayas, Ecuador. Recuperado el 25 de Agosto de 2018, de https://www.eluniverso.com/vida-estilo/2014/06/05/nota/3058246/mortalkombat-cambio-industria-videojuego

ESA. (2018). Industry Facts. Recuperado el 25 de Agosto de 2018, de Entertainment Software Association: http://www.theesa.com/about-esa/industry-facts/

Esnaola, G., y Levis, D. (2009). Videojuegos en redes sociales: aprender desde experiencias óptimas. Revista Internacional de Comunicación Audiovisual, Publicidad y Literatura, 1(7), 265-279. Recuperado el 26 de Agosto de 2018, de https://idus.us.es/xmlui/handle/11441/58316

Facebook. (2018). Facebook para empresas. Recuperado el 1 de Abril de 2018, de Facebook: https://www.facebook.com/business/

Hidalgo, J., Chiriboga, E., y Mora, F. (2015). Estudio de caso: el remozamiento de la identidad visual corporativa del Banco Guayaquil en Ecuador. Razón y Palabra, 19(89), 346-362. Recuperado el 1 de Marzo de 2018, de http://www.revistarazonypalabra.org/index.php/ryp/article/view/246/284

Mercado Libre. (25 de Agosto de 2018). Categorías para comprar y vender. Recuperado el 25 de Agosto de 2018, de https://www.mercadolibre.com.ec/categories.html

Muros, B., Aragón, Y., y Bustos, A. (2013). La ocupación del tiempo libre de jóvenes en el uso de videojuegos y redes. Comunicar, Revista Científica de Educomunicación, XX(40), 31-39. Recuperado el 21 de Diciembre de 2018, de http://dx.doi.org/10.3916/C40-2013-02-03

Núñez, V. (17 de Enero de 2017). Fórmula para calcular engagement y CTR (plantillas). Recuperado el 3 de Enero de 2019, de Vilma Núñez: https://vilmanunez.com/formula-engagement-redes-sociales-ctr/

Paladines, F., Granda, C., \& Velásquez, A. (Junio de 2014). La marca ecuatoriana y su gestión en redes sociales. Razón y Palabra(86). Recuperado el 1 de Abril de 2018, de http://www.redalyc.org/html/1995/199531505033/

Punín, M. I., Martínez, A., \& Rencoret, N. (2014). Medios digitales en Ecuador: perspectivas de futuro. Comunicar, XXI(42), 199-207. Recuperado el 2 de Enero de 2019, de http://dx.doi.org/10.3916/C42-2014-20 
Revuelta, F., \& Bernabé, A. (2012). El videojuego en red social: un nuevo modelo de comunicación. Dialnet(Extra 6), 157-176. Recuperado el 26 de Agosto de 2018, de https://dialnet.unirioja.es/servlet/articulo?codigo $=4026169$

Rodríguez, I. (18 de Junio de 2018). Adicción a videojuegos es enfermedad mental, según la OMS. La Nación. San José, San José, Costa Rica. Recuperado el 25 de Agosto de 2018, de https://www.nacion.com/ciencia/salud/adiccion-a-videojuegos-esenfermedad-mental-segun/NTH5TJL5NZHEVHO3NT4MFG7RP4/story/

Sarmiento, J. (2017). La influencia de la comunicación de marca en la actitud y en el comportamiento de los consumidores a través de los medios sociales. 3C Empresa, 6(4), 12-28. Recuperado el 28 de Diciembre de 2018, de http://dx.doi.org/10.17993/3cemp.2017.060432.12-28

Sinde, J., Medrano, C., y Martinez de Morentin, J. (2015). Transimisión de valores en adolescentes: un análisis con videojuegos. Revista Latina de Comunicación Social(70), 230-251. Recuperado el 21 de Diciembre de 2018, de http://www.revistalatinacs.org/070/paper/1044pv/14es.html

Snead, J. (Productor), Snead, J. (Escritor), \& Snead, J. (Dirección). (2014). Video Games: The Movie [Película]. Estados Unidos. Recuperado el 25 de Agosto de 2018, de https://www.youtube.com/watch?v=YkfWZ4k3eNs

Tur-Viñes, V., y Rodríguez, R. (2014). Transmedialidad, Series de Ficción y Redes Sociales. El Caso de Pulseras Rojas en el Grupo Oficial de Facebook (Antena 3. España). Cuadernos.info(34). Recuperado el 26 de Agosto de 2018, de https://scielo.conicyt.cl/scielo.php?pid=S0719-

367X2014000100010\&script=sci_arttext

Ureña, G. V., Herrera-Murillo, D. J., \& Rodríguez-Martínez, M. d. (Septiembre de 2014). Asociación entre el momento de la publicación en redes sociales y el engagement: estudio de las universidades mexicanas. Palabra Clave, 17(3), 749-772. doi:10.5294/pacla.2014.17.3.8

Veintimilla, E. (5 de Mayo de 2018). El gusto por videojuegos genera millones en ganancias. El Telégrafo. Quito, Pichincha, Ecuador. Recuperado el 25 de Agosto de 2018, de https://www.eltelegrafo.com.ec/noticias/tecnologia/1/el-gusto-porvideojuegos-genera-millones-en-ganancias

Zumárraga, M., Reyes, C., y Carofylis, C. (2017). ¿Verdad o ficción? El uso político de las redes sociales en la participación política offline en las elecciones presidenciales en Ecuador. Análisis Político(91), 130-145. Recuperado el 2 de Enero de 2019, de http://www.scielo.org.co/pdf/anpol/v30n91/0121-4705-anpol30-91-00130.pdf 